\title{
Multimode-Interference Waveguide Crossing Coupled Microring-Resonator-Based Switch Nodes for Photonic Networks-on-Chip
}

\author{
Fang Xu and Andrew W. Poon \\ Photonic Device Laboratory, Department of Electronic and Computer Engineering \\ The Hong Kong University of Science and Technology, Clear Water Bay, Hong Kong SAR, China \\ Tel: (852) 2358 7905; Fax: (852) 2358 1485; e-mail: eeawpoon@ust.hk
}

\begin{abstract}
We propose two-dimensional nonblocking low-power photonic switch nodes for networks-on-chip using multimode-interference-based waveguide crossing-coupled microring electro-optic switch array in silicon-on-insulator.

(C) 2008 Optical Society of America

OCIS codes: (230.5750) Resonators; (060.1810) Switches
\end{abstract}

High-performance multi-core processor design has been facing severe limitations of metal interconnection. In this respect, photonic networks-on-chip using silicon photonics technology offers a potential solution for low latencies and low power consumption which is independent on the data rate [1]. To this end, silicon microring resonator-based electro-optic switch array coupled with waveguide crossings can constitute a potential architecture for on-chip photonic crossbar interconnection. However, one key challenge in realizing such design in silicon is the considerable insertion loss and crosstalk imposed at the high-index-contrast submicrometer-sized waveguide intersections.

Here, we propose a two-dimensional nonblocking crossbar photonic switch node, which comprises an array of four identical silicon microring resonator switches side-coupled with low-loss and low-crosstalk multimode-interference (MMI)-based waveguide crossing [2] array in a silicon-on-insulator (SOI) substrate. Our photonic crossbar design offers low-power dissipation, scalability, and small device foot-print. Our initial experiment of cascaded silicon passive microring resonator coupled MMI crossing array indicates that the proposed architecture is entirely feasible.

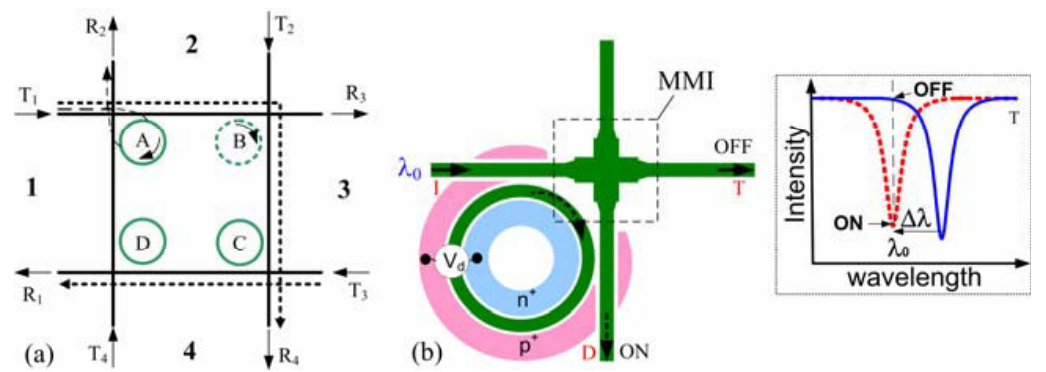

Fig. 1 (a) Proposed two-dimensional nonblocking 4 x 4 crossbar photonic switch node comprising four identical silicon microring electro-optic switches side-coupled to a MMI-based waveguide crossbar. The dashed arrows depict possible light paths between source nodes 1, 2, 3, and 4. T: transmitter, R: receiver. (b) Schematic of a silicon MMI-based waveguide crossing-coupled microring resonator switch with embedded p-i-n diode. Inset: switching scheme at a signal wavelength $\lambda_{0}$. Solid line: "off" state transmission spectrum at port T. Dotted line: "on" state transmission spectrum at port T. $\mathrm{V}_{\mathrm{d}}$ : driving voltage

Figure 1(a) schematically shows our proposed two-dimensional nonblocking 4 x 4 switch node with four identical microring resonators. Each microring resonator acts as an electro-optic switch grid enabled by carrier dispersion effect. Figure 1(b) illustrates the schematic of the single switch grid with an integrated lateral p-i-n diode. We set signal wavelength $\lambda_{0}$ corresponding to an off-resonance wavelength of the microring resonator at off-state (no bias voltage is applied across the $\mathrm{p}-\mathrm{i}-\mathrm{n}$ diode), and also to the blueshifted resonance wavelength of the microresonator at on-state (upon forward biasing the p-i-n diode). Thus, the optical signal at $\lambda_{0}$ wavelength launched from port I of the horizontal waveguide is transmitted to port T when the switch is off, and is routed to the vertical port $\mathrm{D}$ when the switch is on.

This 4 x 4 switch node can be controlled by a simple routing algorithm which permits arbitrary one-to-one 


\section{CWP4.pdf}

interconnection between four source nodes. We see that no more than one microring switch needs to be switched on for establishing any single light path (except for self-communication). Besides, communications between two source nodes connected in the same direction (e.g. nodes 1 and 3) consume no switching power. Assuming a microring with resonance $\mathrm{Q}$ of $\sim 10^{4}$, we see that a minimum resonance wavelength shift for the switching is $\sim 0.15 \mathrm{~nm}$ in the $1550 \mathrm{~nm}$ wavelengths. Such spectral shift can be induced by carrier injection of $1 \times 10^{17} \mathrm{~cm}^{-3}$ (assuming the p-i-n diode surrounds $3 / 4$ of the microring), suggesting low-current requirement of $\sim 40 \mathrm{nA}$ at $\mathrm{V}_{\mathrm{d}} \sim 0.8 \mathrm{~V}$ and thereby potentially low-power consumption of $\sim 32 \mathrm{nW}$ according to our semiconductor device simulations using MEDICI (assuming $\mathrm{p}^{+}: 2 \times 10^{19} \mathrm{~cm}^{-3}, \mathrm{n}^{+}: 10^{20} \mathrm{~cm}^{-3}$, intrinsic region width $=1.05 \mu \mathrm{m})$. We remark that our previous work on $10-\mu \mathrm{m}$-diameter silicon microdisk resonator switch with embedded lateral p-i-n diode [3] suggested that in practice a $\sim 20 \mu \mathrm{W}$ power dissipation may be required (in part due to current leakage) for a $0.06 \mathrm{~nm}$ wavelength tuning. We also expect that our switch node should have a switching speed on the order of ns - sub-ns [3,4] using carrier-injection-based electro-optic effect, and therefore suitable for packet switching. Another important advantage of the microring crossbar switch is the compact

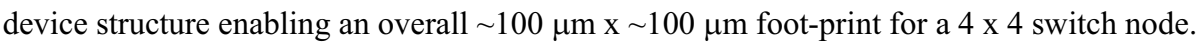
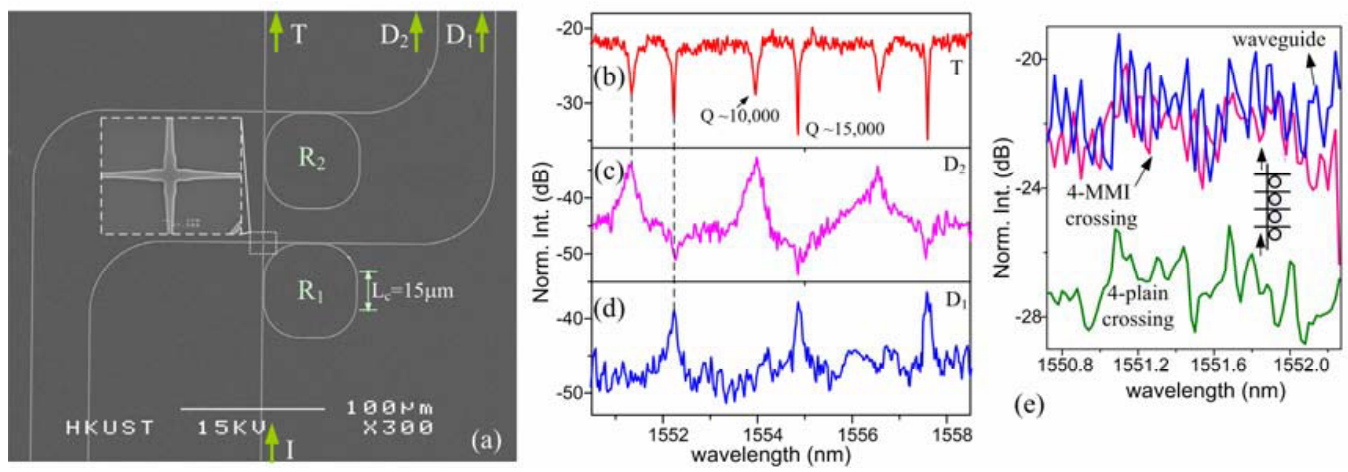

Fig. 2. (a) Scanning electron micrograph of the fabricated two-channel microring-resonator-coupled MMI crossing filter in SOI. The square-shaped microring resonators have four curved waveguides with radii $r_{l, 2}$ and four straight waveguides with interaction length $L_{c}=15 \mu \mathrm{m}$. The waveguide width is $0.4 \mu \mathrm{m} . \quad r_{I}=25 \mu \mathrm{m}$ for microring $\mathrm{R}_{1}$ and $r_{2}=25.5 \mu \mathrm{m}$ for microring $\mathrm{R}_{2}$. $\mathrm{I}$ : input, $\mathrm{T}$ : throughput, $\mathrm{D}_{1}, \mathrm{D}_{2}$ : drop. (b) Measured TE-polarized throughput-port transmission spectrum at port T. (c), (d) Measured drop-port transmission spectra at ports (c) $D_{2}$, and (d) $D_{1}$. (e) Measured transmission spectra for four cascaded microring-coupled MMI-based crossing (pink) and controlled four cascaded microring-coupled plain crossing (green) at off-resonance wavelengths. The blue-line spectrum shows the controlled single waveguide transmission.

As a proof-of-concept, Fig. 2 (a) shows the scanning electron micrograph of the fabricated device. The inset shows a zoom-in view of the MMI crossing design according to our previous work [2]. Figure 2(b) shows the measured TE-polarized throughput-port transmission spectrum and the two drop-port transmission spectra. We see the Q-factor exceeds $10^{4}$ and the extinction ratio exceeds $10 \mathrm{~dB}$. Figure $2(\mathrm{e})$ shows the measured transmission spectra for four cascaded MMI-based and controlled plain crossing coupled microring resonators at off-resonance wavelengths. We attribute the modulations to multiple-interference of the waveguide end-face reflections. The four cascaded MMI crossings induce in average an extra loss of $\sim 1 \mathrm{~dB}$ compared with the controlled waveguide of the same width $\sim 0.4 \mu \mathrm{m}$. This represents in average a $\sim 4 \mathrm{~dB}$ loss improvement compared with the controlled cascaded plain crossings which is consistent with our earlier work [2]. Hence, based on our previous work [2] and [3], we see that it is entirely feasible to realize our proposed switch node. Further design and fabrication of the proposed cross-connect switch node using lateral p-i-n diode integrated microring resonators are in progress.

[1] A. Shacham, K. Bergman, and L. P. Carloni, "On the design of a photonic network-on-chip," in proceeding of the First International Symposium on Networks-on-Chip, Nocs'07, pp.53-64, 2007.

[2] H. Chen and A. W. Poon, "Low-loss multimode-interference-based crossings for silicon wire waveguides," IEEE Photon. Technol. Lett., 18, 2260-2262 (2006).

[3] L. Zhou and A. W. Poon, "Silicon electro-optic modulators using p-i-n diodes embedded 10-micron-diameter microdisk resonators," Opt. Express, 14, 6851-6857 (2006).

[4] Q. Xu, S. Manipatruni, B. Schmidt, J. Shakya, and M. Lipson, "12.5 Gbit/s carrier-injection-based silicon micro-ring silicon modulators," Opt. Express, 15, 430-436 (2007). 